Research Paper

\title{
Value of Sarcopenia defined by the new EWGSOP2 consensus for the prediction of Postoperative Complications and Long-term Survival after Radical Gastrectomy for Gastric Cancer: A comparison with four common nutritional screening tools
}

\author{
Dong-Dong Huang ${ }^{1 *}$, Hui-Yang Cai ${ }^{*}$, Xi-Yi Chen², Wen-Xi Dong ${ }^{1}$, Drugyel Wangchuk ${ }^{1}$, Jing-Yi Yan ${ }^{1}$, Xiao-Lei \\ Chen $^{1 \bowtie}$ and Qian-Tong Dong ${ }^{1 凶}$ \\ 1. Department of Gastrointestinal Surgery, The First Affiliated Hospital of Wenzhou Medical University, Wenzhou, China. \\ 2. Department of Cardiothoracic Surgery, The Second Affiliated Hospital of Wenzhou Medical University, Wenzhou, China. \\ *These authors contributed equally to this study. \\ $\triangle$ Corresponding authors: E-mail: dongqiantong0577@163.com (Qian-Tong Dong), chenxiaolei@wzhospital.cn (Xiao-Lei Chen); Department of Gastrointestinal Surgery, \\ The First Affiliated Hospital of Wenzhou Medical University, 2 Fuxue Lane, Wenzhou 325000, Zhejiang, China. \\ (C) The author(s). This is an open access article distributed under the terms of the Creative Commons Attribution License (https://creativecommons.org/licenses/by/4.0/). \\ See http:/ /ivyspring.com/terms for full terms and conditions.
}

Received: 2020.06.22; Accepted: 2020.07.27; Published: 2020.08.06

\begin{abstract}
Background: Nutritional risk and sarcopenia are both associated with increased postoperative morbidity and mortality following elective surgery. This study aimed to investigate whether sarcopenia has additional predictive value for postoperative complications and long-term survival besides nutritional screening tools.

Methods: Clinical data of patients underwent radical gastrectomy for gastric cancer was prospectively collected. Sarcopenia was diagnosed by grip strength plus muscle quanlity/quality based on preoperative abdominal CT scans. Nutritional screening was performed using 4 common nutritional screening tools, including Malnutrition Universal Screening Tool (MUST), Nutritional Risk Screening (NRS)-2002, Malnutrition Screening Tool (MST), and Short Nutritional Assessment Questionnaire (SNAQ).

Results: A total of 880 patients were analyzed, in which 167 (18.98\%) were diagnosed with sarcopenia. The incidence of nutritional risk identified by the 4 tools were $44.66 \%$ (MUST $\geq 1$ ), $35.23 \%$ (NRS-2002 $\geq 3$ ), $29.89 \%$ (MST $\geq 2$ ), and $20.34 \%$ (SNAQ $\geq 2$ ). Multivariate analyses showed that nutritional risk identified by the 4 nutritional screening tools were not independently associated with postoperative complications, overall survival (OS) or disease-free survival (DFS), except for NRS-2002 $\geq 3$ as an independent risk factor of OS. Sarcopenia was always an independent risk factor for postoperative complications, OS, and DFS after adjusting for nutritional risk and the other covariates in the multivariate analyses.

Conclusions: MUST, NRS-2002, MST, and SNAQ had low predictive power for postoperative complications and long-term survival in patients underwent radical gastrectomy for gastric cancer. Sarcopenia had additional predictive value for postoperative complications and long-term survival besides these nutritional screening tools and should be implemented in the preoperative assessments.
\end{abstract}

Key words: Sarcopenia; Malnutrition; Gastrectomy; Postoperative complication; Survival

\section{Introduction}

In recent years, sarcopenia has been increasingly recognized as a strong predictor for morbidity and mortality after surgery [1-3]. According to the revised European consensus on definition and diagnosis of sarcopenia recommended by the European Working
Group on Sarcopenia in Older People 2 (EWGSOP2), sarcopenia is diagnosed based on the measurement of muscle strength plus muscle quality/quantity [4]. Computed tomography (CT) is considered to be a gold standard for the assessment of muscle mass and 
attenuation [4]. Moreover, preoperative abdominal CT scan is necessary for patients with gastrointestinal tumors for staging of the tumor and guidance of surgery. However, muscle mass measurement before surgery is not a routine practice for radiologists in most medical centers. Therefore, sarcopenia has not been widely used in clinical practice for preoperative assessment. A recent study showed that CT measured sarcopenia may have only little additional value over Malnutrition Universal Screening Tool (MUST) for the prediction of postoperative complications after colorectal cancer surgery [5]. This finding aroused us to speculate whether measurement of sarcopenia is still necessary for preoperative risk assessment in the context of nutritional risk screening before surgery for other types of malignancy, such as gastric cancer.

Malnutrition is present in about $30 \%$ of hospitalized patients, and the prevalence is especially high in patients with gastrointestinal tumors, such as gastric cancer [6,7]. There is convincing evidence that nutritional risk is associated with increased postoperative morbidity and mortality following elective surgery $[8,9]$. In contrast to the limited application of sarcopenia assessment in clinical practice, screening of nutritional risk has been applied in many medical centers world-widely. Therefore, it is worthwhile to investigate whether sarcopenia has additional predictive value besides nutritional screening tools for prediction of surgical prognosis when the nutritional screening has already been well implemented. Besides, although there are many nutritional screening tools, such Malnutrition Universal Screening Tool (MUST) [10], Nutrition Risk Screening 2002 (NRS-2002) [11], Malnutrition Screening Tool (MST) [12], and Short Nutritional Assessment Questionnaire (SNAQ) [13], it has as yet not been established which is the optimal nutritional assessment tool in patients scheduled to receive gastrectomy for gastric cancer.

In the present study, we aimed to investigate whether sarcopenia has additional predictive value besides 4 common nutritional screening tools (MUST, NRS-2002, MST, and SNAQ) for the prediction of postoperative complications and long-term survival after radical gastrctomy for gastric cancer.

\section{Methods}

\section{Patients}

From August 2014 to February 2018, patients who underwent radical gastrectomy for gastric cancer at the Gastrointestinal Surgery Department of the First Hospital Affiliated to Wenzhou Medical University were included in this prospective study. The inclusion criteria included patients who (1) were aged 18 years old or more; (2) had American Society of Anesthesiologists (ASA) grade SIII; (3) planned to receive elective gastrectomy for gastric cancer with curative intent; (4) had preoperative abdominal CT images available for review (no more than 1 month before operation); and (5) agreed to take part in this study and signed the informed consent. Exclusion criteria included patients who (1) were aged $<18$ years; (2) were unable to be assessed for nutritional status due to mental disorder or disturbance of consciousness; (3) were unable be measured for muscle strength or physical performance due to physical deformity; (4) declined to take part in the study; and (5) underwent palliative operation. This project was approved by the Ethical Review Board of The First Affiliated Hospital of Wenzhou Medical University and registered in China Clinical Trial Registry (NO. ChiCTR1800019717). All patients received standard managements and treatments following the Japanese gastric cancer treatment guidelines 2010 (version 3) [14].

\section{Data collection}

The following data were collected prospectively: (1) preoperative patient and disease characteristics, including age, gender, body mass index (BMI), plasma albumin concentration, haemoglobin concentration, ASA grade, comorbidity, tumor-nodemetastasis (TNM) stage of tumor, differentiation of tumor, tumor location, previous abdominal surgery; (2) operative and treatment details, including laparoscopy-assisted surgery, type of resection, extent of lymph node dissection, combined organ resection, adjuvant chemotherapy; and (3) postoperative outcomes, including postoperative complications and mortality. Only complications classified as Grade II or above according to the Clavien-Dindo classification were analyzed in this study [15], and complications classified as Grade III or above were identified as severe complications.

\section{Diagnosis of sarcopenia}

According to the revised European consensus on definition and diagnosis of sarcopenia recommended by EWGSOP2, sarcopenia was defined by low muscle strength plus low muscle quantity or quality. Severe sarcopenia was defined when the sarcopenic patients had additional low physical performance [4]. Muscle quantity was measured using cross sectional CT images at the third lumbar vertebra (L3) level on the first image with both vertebral spines visible [16]. Skeletal muscle tissues were identified by the Hounsfield units (HU) with the thresholds range of -29 to +150, using the image processing system (version 3.0.11.3 BN17 32 bit; INFINITT Healthcare 
Co., Ltd). Cross-sectional area of skeletal muscle tissue was normalized by the height squared to calculate the skeletal muscle index $\left(\mathrm{SMI}, \mathrm{cm}^{2} / \mathrm{m}^{2}\right)$. Patients with L3 skeletal muscle index $<40.8 \mathrm{~cm}^{2} / \mathrm{m}^{2}$ for men and $<34.9 \mathrm{~cm}^{2} / \mathrm{m}^{2}$ for women were identified as having low muscle mass [1]. Muscle quality was measured by using mean muscle attenuation. The sex-specific cutoff values for defining low muscle attenuation were $28.6 \mathrm{HU}$ for females and $38.5 \mathrm{HU}$ for males according to our previous study [17]. All patients received measurements of handgrip strength and 6-m usual gait speed tests in the first hospitalized day before surgery. Muscle strength was determined by handgrip strength tests, using an electronic hand dynamometer (EH101; CAMRY, Guangdong Province, China). Patients with handgrip strength $<26$ $\mathrm{kg}$ for men and $<18 \mathrm{~kg}$ for women were identified as having low muscle strength. Physical performance was determined by 6-m usual gait speed tests as described previously $[1,3]$. Patients with 6-m usual gait speed $<0.8 \mathrm{~m} / \mathrm{s}$ were identified as having low physical performance.

\section{Nutritional screening}

All patients received nutritional screening using 4 common nutritional screening tools, including Malnutrition Universal Screening Tool (MUST) [10], Nutrition Risk Screening 2002 (NRS-2002) [11], Malnutrition Screening Tool (MST) [12], and Short Nutritional Assessment Questionnaire (SNAQ) [13]. The MUST scores are based on BMI, unplanned weight loss, and reduced food intake for more than 5 days due to the effect of acute diseases. Patients with an MUST score of $\geq 1$ are classified as being at nutritional risk [10]. NRS-2002 identify the risk of malnutrition based on 5 variables, including general condition, BMI, weight loss, food intake over the last week, and the patients' age. Patients with an NRS 2002 score of $\geq 3$ are classified as being at nutritional risk [11]. The MST tool is based on unintentional weight loss, the extent of weight loss, and reduced food intake due to decreased appetite. Patients with an MST score of $\geq 2$ are classified as being at nutritional risk [12]. The SNAQ tool is based on unintentional weight loss, decreased appetite over the last month, and usage of supplemental drinks or tube feeding over the last month. Patients with an SNAQ score of $\geq 2$ are classified as being at nutritional risk [13].

\section{Follow up}

All patients were routinely followed up within the first month after surgery in the outpatient. After that, patients were followed up every 3 months for 2 years and every 6 months thereafter by telephone interviews or outpatient visits. The follow-up program was consisted of physical examination, laboratory tests, and ultrasonography and/or CT and/or endoscopy. The last follow-up date was January 2019. Overall survival (OS) was defined as the period from the time of surgery to the time of death due to any causes. Disease-free survival (DFS) was calculated from the date of surgery to the date of relapse or death due to cancer-free causes.

\section{Statistical analysis}

Normally distributed data were presented as mean and standard deviation (SD) and compared using the t-test. Nonnormally distributed data were presented as median and interquartile range (IQR) and compared using the Mann-Whitney U-test. Categorical data were compared using the chi-square test or Fisher exact test. Univariate analysis was used to identify potential risk factors for postoperative complications, overall survival and disease-free survival. Multivariate logistic regression analysis or Cox proportional-hazards analysis included variables with a P-value of $<0.10$ in the univariate analysis, as well as other clinical relevant variables, including age, gender, BMI or adjuvant chemotherapy. Survival curves were estimated using the Kaplan-Meier method and compared using log-rank tests. All data were analyzed using SPSS statistics version 22.0 (IBM, USA). Two-tailed $\mathrm{P}$ values $<0.05$ were considered statistically significant.

\section{Results}

From August 2014 to February 2018, 948 patients who underwent gastrectomy for gastric cancer met our inclusion criteria initially. Sixty-eight patients were excluded for reasons of underwent palliative surgery, unable to be assessed for muscle strength or physical performance, failed to be screened for nutritional status or refused to participate in the study. Finally, a total of 880 patients were included in the analyses.

Sarcopenia was identified in $167(18.98 \%)$ of the patients, in which $53(6.02 \%)$ patients were identified as having severe sarcopenia. Compared with nonsarcopenic patients, patients with sarcopenia had older age, higher ASA grade, higher TNM stage, lower BMI, lower L3 SMI, lower muscle attenuation, lower handgrip strength, lower gait speed, lower preoperative albumin and hemoglobin levels, and received less laparoscopy-assisted surgery (Table 1). The incidence of nutritional risk identified by the 4 nutritional screening tools in our patients were $44.66 \%$ (MUST $\geq 1$ ), 35.23\% (NRS-2002 $\geq 3$ ), 29.89\% (MST $\geq 2$ ), and $20.34 \%$ (SNAQ $\geq 2$ ). Sarcopenia showed a significant correlation with nutritional risk identified 
by all of 4 nutritional screening tools (Table 1). However, the agreement of sarcopenia with nutritional risk is slight (kappa $<0.2)$.

Table 1. Patient demographic and clinical characteristics

\begin{tabular}{|c|c|c|c|c|}
\hline Factors & All $(n=880)^{a}$ & $\begin{array}{l}\text { Nonsarcopenic } \\
(\mathrm{n}=713)^{\mathrm{a}}\end{array}$ & $\begin{array}{l}\text { Sarcopenic } \\
(\mathrm{n}=167)^{\mathrm{a}}\end{array}$ & $P$ \\
\hline Age, mean (SD), years & $64.47(10.80)$ & $62.35(10.35)$ & $73.54(7.50)$ & $<0.001^{*}$ \\
\hline Gender & & & & 0.645 \\
\hline Female & 230 & 184 & 46 & \\
\hline Male & 650 & 529 & 121 & \\
\hline BMI, mean (SD), kg/m² & $22.63(3.07)$ & $22.91(3.04)$ & $21.48(2.96)$ & $<0.001^{*}$ \\
\hline NRS-2002 scores & & & & $<0.001^{*}$ \\
\hline$<3$ & 570 & 496 & 74 & \\
\hline$\geq 3$ & 310 & 217 & 93 & \\
\hline MUST scores & & & & $<0.001^{*}$ \\
\hline$<1$ & 487 & 419 & 68 & \\
\hline$\geq 1$ & 393 & 294 & 99 & \\
\hline MST scores & & & & $0.003^{*}$ \\
\hline$<2$ & 617 & 516 & 101 & \\
\hline$\geq 2$ & 263 & 197 & 66 & \\
\hline SNAQ scores & & & & $0.005^{*}$ \\
\hline$<2$ & 701 & 581 & 120 & \\
\hline$\geq 2$ & 179 & 132 & 47 & \\
\hline L3 SMI, mean (SD), $\mathrm{cm}^{2} / \mathrm{m}^{2}$ & $42.73(7.74)$ & $43.86(7.60)$ & $37.89(6.41)$ & $<0.001^{*}$ \\
\hline $\begin{array}{l}\text { Muscle attenuation, } \\
\text { mean (SD), HU }\end{array}$ & $36.12(7.81)$ & 37.43 (7.39) & 30.54 (7.10) & $<0.001^{*}$ \\
\hline $\begin{array}{l}\text { Handgrip strength, } \\
\text { mean (SD), kg }\end{array}$ & $28.40(9.22)$ & $30.84(8.21)$ & $18.11(5.25)$ & $<0.001^{*}$ \\
\hline $\begin{array}{l}\text { Gait speed, } \\
\text { median (IQR), m/s }\end{array}$ & $1.00(0.27)$ & $1.03(0.25)$ & $0.81(0.28)$ & $<0.001^{*}$ \\
\hline Albumin, mean (SD), $\mathrm{g} / \mathrm{L}$ & $38.11(4.45)$ & $38.71(4.16)$ & $35.51(4.74)$ & $<0.001^{*}$ \\
\hline $\begin{array}{l}\text { Hemoglobin, } \\
\text { mean (SD), g/L }\end{array}$ & $\begin{array}{l}120.97 \\
(21.61)\end{array}$ & $123.52(21.16)$ & $\begin{array}{l}110.08 \\
(20.15)\end{array}$ & $<0.001^{*}$ \\
\hline ASA grade & & & & $<0.001^{*}$ \\
\hline I & 208 & 183 & 25 & \\
\hline II & 522 & 426 & 96 & \\
\hline III & 150 & 104 & 46 & \\
\hline Charlson comorbidity index & & & & 0.088 \\
\hline 0 & 622 & 513 & 109 & \\
\hline 1 & 174 & 139 & 35 & \\
\hline$\geq 2$ & 84 & 61 & 23 & \\
\hline Previous abdominal surgery & & & & 0.145 \\
\hline No & 777 & 635 & 142 & \\
\hline Yes & 103 & 78 & 25 & \\
\hline Tumor location & & & & 0.579 \\
\hline Not at cardia & 765 & 622 & 143 & \\
\hline At cardia & 115 & 91 & 24 & \\
\hline Differentiation of tumor & & & & 0.505 \\
\hline Well differentiated & 304 & 250 & 54 & \\
\hline Poorly differentiated & 576 & 463 & 113 & \\
\hline TNM stage & & & & $0.001^{*}$ \\
\hline I & 310 & 272 & 38 & \\
\hline II & 214 & 167 & 47 & \\
\hline III & 356 & 274 & 82 & \\
\hline \multicolumn{3}{|c|}{ Extent of lymph node dissection } & & 0.219 \\
\hline D1 & 115 & 98 & 17 & \\
\hline D2 & 765 & 615 & 150 & \\
\hline Type of resection & & & & 0.103 \\
\hline Subtotal gastrectomy & 549 & 454 & 95 & \\
\hline Total gastrectomy & 331 & 259 & 72 & \\
\hline Combined resection & & & & 0.069 \\
\hline No & 810 & 662 & 148 & \\
\hline Yes & 70 & 51 & 19 & \\
\hline \multicolumn{3}{|c|}{ Laparoscopy-assisted surgery } & & $0.001^{*}$ \\
\hline No & 644 & 504 & 140 & \\
\hline Yes & 236 & 209 & 27 & \\
\hline
\end{tabular}

Table 2. Univariate analysis for risk factors associated with postoperative complications

\begin{tabular}{|c|c|c|c|}
\hline & $\begin{array}{l}\text { Without complications } \\
(\mathrm{n}=649)\end{array}$ & $\begin{array}{l}\text { With complications } \\
(\mathrm{n}=231)\end{array}$ & $P$ \\
\hline \multicolumn{3}{|c|}{ (2: } & $0.022^{*}$ \\
\hline$<1$ & 374 & 113 & \\
\hline$\geq 1$ & 275 & 118 & \\
\hline \multicolumn{3}{|l|}{ NRS-2002 } & $0.001^{*}$ \\
\hline$<3$ & 441 & 129 & \\
\hline$\geq 3$ & 208 & 102 & \\
\hline \multicolumn{3}{|l|}{ MST } & 0.067 \\
\hline$<2$ & 466 & 151 & \\
\hline$\geq 2$ & 183 & 80 & \\
\hline \multicolumn{3}{|l|}{ SNAQ } & $0.004^{*}$ \\
\hline$<2$ & 532 & 169 & \\
\hline$\geq 2$ & 117 & 62 & \\
\hline \multicolumn{3}{|l|}{ Sarcopenia } & $<0.001^{*}$ \\
\hline No & 560 & 153 & \\
\hline Yes & 89 & 78 & \\
\hline \multicolumn{3}{|l|}{ Gender } & 0.144 \\
\hline Female & 178 & 52 & \\
\hline Male & 471 & 179 & \\
\hline \multicolumn{3}{|l|}{ Age } & $<0.001^{*}$ \\
\hline$<75 \mathrm{y}$ & 555 & 166 & \\
\hline$\geq 75 \mathrm{y}$ & 94 & 65 & \\
\hline \multicolumn{3}{|l|}{ BMI } & 0.285 \\
\hline$\geq 18.5$ & 604 & 210 & \\
\hline$<18.5$ & 45 & 21 & \\
\hline \multicolumn{3}{|l|}{ ASA } & $0.001^{*}$ \\
\hline I, II & 555 & 175 & \\
\hline III & 94 & 56 & \\
\hline \multicolumn{3}{|c|}{ Charlson comorbidity index } & $<0.001^{*}$ \\
\hline 0 & 482 & 140 & \\
\hline$\geq 1$ & 167 & 91 & \\
\hline \multicolumn{3}{|l|}{ Anemia } & $<0.001^{*}$ \\
\hline No & 439 & 117 & \\
\hline Yes & 210 & 114 & \\
\hline \multicolumn{3}{|l|}{ Hypoproteinemia } & $<0.001^{*}$ \\
\hline No & 522 & 155 & \\
\hline Yes & 127 & 76 & \\
\hline \multicolumn{3}{|c|}{ Previous abdominal surgery } & 0.155 \\
\hline No & 579 & 198 & \\
\hline Yes & 70 & 33 & \\
\hline \multicolumn{3}{|l|}{ TNM stage } & 0.188 \\
\hline I & 240 & 70 & \\
\hline II & 153 & 61 & \\
\hline III & 256 & 100 & \\
\hline \multicolumn{3}{|l|}{ Tumor differentiation } & 0.974 \\
\hline Differentiated & 224 & 80 & \\
\hline Undifferentiated & 425 & 151 & \\
\hline \multicolumn{3}{|l|}{ Tumor location } & $0.007^{*}$ \\
\hline Not cardia & 576 & 189 & \\
\hline Cardia & 73 & 42 & \\
\hline Type of resection & & & 0.110 \\
\hline Subtotal gastrectomy & 415 & 134 & \\
\hline Total gastrectomy & 234 & 97 & \\
\hline Laparoscopic surgery & & & $0.010^{*}$ \\
\hline No & 460 & 184 & \\
\hline Yes & 189 & 47 & \\
\hline Combined organ resec & & & 0.305 \\
\hline No & 601 & 209 & \\
\hline Yes & 48 & 22 & \\
\hline Extent of lymph node & section & & 0.619 \\
\hline D1 & 87 & 28 & \\
\hline D2 & 562 & 203 & \\
\hline
\end{tabular}

In the 880 included patients, 231 (26.25\%) occurred postoperative complications graded II and above (Table 2). The details of postoperative 
complications were listed in Table S1. Univariate analyses showed that MUST $\geq 1$, NRS-2002 $\geq 3$, SNAQ $\geq 2$, sarcopenia, age $\geq 75$ years, ASA $=I I I$, Charlson comorbidity index $\geq 1$, anemia, hypoproteinemia, tumor located at cardia, and laparoscopic surgery were significantly associated with postoperative complications (Table 2). None of the 4 nutritional screening tools had good sensitivity for the prediction of postoperative complications, ranging from $26.84 \%$ to $51.08 \%$ (Table S2). Sarcopenia had a high specificity $(86.29 \%)$ but low sensitivity $(33.77 \%)$ for the prediction of postoperative complications (Table S2). To further compare the predictive values of the 4 nutritional screening tools on the postoperative complications, we performed 4 separate multivariate analyses, adjusting the effect of the 4 nutritional screening tools for the same covariates, including gender, age, BMI, Charlson comorbidity index, anemia, hypoproteinemia, tumor location, laparoscopic surgery, and sarcopenia. None of these nutritional screening tools remained independently associated with postoperative complications after adjusting for these covariates in the multivariate analyses. Sarcopenia, tumor located at cardia, and Charlson comorbidity index $\geq 1$ were the independent risk factors for postoperative complications in all of the 4 multivariate analyses (Table 3).

Univariate analyses and Kaplan-Meier analyses showed that MUST $\geq 1$, MST $\geq 2$, SNAQ $\geq 2$, and NRS-2002 $\geq 3$ were all associated with worse OS (Table 4, Figure S1). However, in the multivariate analyses, only NRS-2002 $\geq 3$ remained an independent risk factor for OS. Other independent risk factors for OS were sarcopenia, higher TNM stage, undifferentiated tumor histological type, total gastrectomy in all of the 4 multivariate analyses, whereas adjuvant chemotherapy was an independent protective factor (Table 5). Univariate analyses and Kaplan-Meier analyses showed that MUST $\geq 1$, MST $\geq 2$ and SNAQ $\geq 2$ were associated with worse DFS (Table 4, Figure S1). However, none of these nutritional screening tools remained significant in the multivariate analyses for DFS. Sarcopenia, higher TNM stage, undifferentiated tumor histological type, total gastrectomy were independently associated the worse DFS in all of the 4 multivariate analyses, whereas adjuvant chemotherapy was an independent protective factor (Table 6).

\section{Discussion}

The 4 nutritional screening tools in the present study were initially developed to screen the nutritional risk. MUST was developed by the Malnutrition Advisory Group for application to all adult patients across all health care settings. It is supported by many governmental and nongovernmental organizations and is the commonly used globally, especially in the European countries [10]. NRS-2002 was created on the basis of its power to predict the therapeutic effect of nutritional support [11], and was recommend by ESPEN for the screening of nutritional risk in hospitalized patients [6]. MST was developed based on its predictive ability of subjective global assessment (SGA) in mixed hospital patient population [12]. SNAQ was created based on its predictive values for nutritional status in patients in the mixed internal and surgery/oncology wards [13]. In the present study, we compared the 4 nutritional screening tools for their predictive value for postoperative outcomes after gastrectomy for gastric cancer. We found that most of these nutritional screening tools showed significant association with postoperative complications, OS, and DFS, suggesting the close relationship between nutritional status and postoperative outcomes. However, none of these tools showed a good sensitivity for the prediction of postoperative complications, ranging from $26.84 \%$ to $51.08 \%$. Receiver operating characteristic (ROC) curves showed a low predictive power of postoperative complications for the 4 tools, ranging from 0.532 to 0.561 (Table S2). Moreover, none of these tools were independent risk factors for postoperative complications or long-term survival in the multivariate analyses, except for NRS2002 as an independent risk factor for overall survival. This indicated that NRS-2002 had a superior predictive value for postoperative survival over the other 3 nutritional screening tools. Based on the above findings, we suggested that nutritional screening is not enough for surgical risk assessment in patients underwent radical gastrectomy for gastric cancer.

In the multivariate analyses, sarcopenia always remained an independent risk factor for postoperative complications and long-term survival after adjusting for nutritional risk determined by each of these nutritional screening tools and the other covariates. This result strongly demonstrated the additional predictive value of sarcopenia on postoperative complications and long-term survival over all of these nutritional screening tools. One of our recent study showed that sarcopenia was an independent risk factor for postoperative complications after gastrectomy in patients without nutritional risk [18], which also demonstrated the additional predictive value of sarcopenia over the nutritional screening tools.

Few studies have compared the value of sarcopenia with nutritional screening tools for the prediction of postoperative outcomes. A recent study by Kroft et al. showed sarcopenia measured by CT 
have only little additional value over the MUST for the prediction of postoperative morbidity after colorectal cancer surgery, which was in contrast with our findings [5]. The reason of the inconsistent results can be explained by the following factors. First, in the study of Kroft et al., sarcopenia was diagnosed by measuring skeletal muscle quantity/quality only. However, the functional aspects of sarcopenia, including muscle strength and physical performance, are also significant for the prediction of postoperative outcomes [1,3]. Second, the study of Kroft et al. was based on patients underwent colorectal cancer surgery, which has certain differences with our patient underwent gastrectomy for gastric cancer. However, the present study and the previous study by Kroft et al. are all single-center studies including patients underwent surgery for homogeneous types of malignancy. It is unknown whether the predictive value of sarcopenia besides nutritional risk is dependent on certain types of surgery or universal among general major abdominal surgeries. Therefore, we suggest that future multi-center studies including patients with various types of malignancy were needed to further demonstrate the predictive value of sarcopenia besides nutritional risk for the postoperative outcomes.

Table 3. Multivariate analysis for risk factors associated with postoperative complications

\begin{tabular}{|c|c|c|c|c|c|c|c|c|}
\hline & MUST $\geq 1$ & & NRS-2002 $\geq 3$ & & $\mathrm{MST} \geq 2$ & & SNAQ $\geq 2$ & \\
\hline & OR $(95 \% \mathrm{CI})$ & $P$ & OR $(95 \% \mathrm{CI})$ & $P$ & OR $(95 \% \mathrm{CI})$ & $P$ & OR $(95 \% \mathrm{CI})$ & $P$ \\
\hline Nutritional risk & $1.172(0.834-1.647)$ & 0.361 & $1.198(0.847-1.694)$ & 0.308 & $1.098(0.775-1.555)$ & 0.600 & $1.417(0.968-2.073)$ & 0.073 \\
\hline Sarcopenia & $2.270(1.523-3.383)$ & $<0.001^{*}$ & $2.252(1.509-3.360)$ & $<0.001^{*}$ & $2.288(1.536-3.410)$ & $<0.001^{*}$ & $2.270(1.522-3.385)$ & $<0.001^{*}$ \\
\hline Gender (male/female) & $1.247(0.856-1.818)$ & 0.251 & $1.245(0.855-1.815)$ & 0.253 & $1.246(0.853-1.820)$ & 0.255 & $1.256(0.862-1.830)$ & 0.236 \\
\hline Age $\geq 75 y$ & $1.443(0.962-2.165)$ & 0.076 & $1.412(0.940-2.123)$ & 0.097 & $1.430(0.952-2.146)$ & 0.085 & $1.455(0.968-2.187)$ & 0.071 \\
\hline BMI $<18.5$ & $0.971(0.524-1.799)$ & 0.926 & $0.987(0.539-1.807)$ & 0.966 & $1.035(0.570-1.879)$ & 0.910 & $1.016(0.559-1.847)$ & 0.958 \\
\hline Charlson comorbidity index $\geq 1$ & $1.838(1.319-2.562)$ & $<0.001^{*}$ & $1.823(1.308-2.542)$ & $<0.001^{*}$ & $1.821(1.306-2.540)$ & $<0.001^{*}$ & 1.819 (1.304-2.537) & $<0.001^{*}$ \\
\hline Anemia & $1.407(0.979-2.021)$ & 0.065 & $1.422(0.991-2.039)$ & 0.056 & $1.426(0.994-2.046)$ & $0.054^{*}$ & $1.399(0.974-2.008)$ & 0.069 \\
\hline Hypoproteinemia & $1.243(0.827-1.869)$ & 0.295 & $1.225(0.813-1.846)$ & 0.333 & $1.251(0.833-1.881)$ & 0.280 & $1.219(0.809-1.835)$ & 0.344 \\
\hline Tumor located at cardia & $1.784(1.154-2.756)$ & $0.009^{*}$ & $1.759(1.138-2.719)$ & $0.011^{*}$ & $1.777(1.150-2.746)$ & $0.010^{*}$ & $1.791(1.158-2.769)$ & $0.009^{*}$ \\
\hline Laparoscopic surgery & $0.785(0.536-1.151)$ & 0.215 & $0.790(0.538-1.159)$ & 0.277 & $0.780(0.533-1.144)$ & 0.203 & $0.787(0.537-1.154)$ & 0.220 \\
\hline
\end{tabular}

*Statistically significant $(P<0.05)$;

OR, odds ratio; $\mathrm{CI}$, confidence interval; BMI, body mass index.

Table 4. Univariate analysis for risk factors associated with overall survival and disease-free survival

\begin{tabular}{|c|c|c|c|c|}
\hline & \multicolumn{2}{|l|}{ Overall survival } & \multicolumn{2}{|l|}{ Disease-free survival } \\
\hline & HR $(95 \%$ CI $)$ & $P$ & $\operatorname{HR}(95 \% \mathrm{CI})$ & $P$ \\
\hline MUST $\geq 1$ & $1.765(1.333-2.336)$ & $<0.001^{*}$ & $1.474(1.097-1.982)$ & $0.010^{*}$ \\
\hline NRS-2002 $\geq 3$ & $1.884(1.427-2.489)$ & $<0.001^{*}$ & $1.269(0.937-1.719)$ & 0.124 \\
\hline MST $\geq 2$ & $1.632(1.226-2.173)$ & $0.001^{*}$ & $1.446(1.061-1.971)$ & $0.020^{*}$ \\
\hline SNAQ $\geq 2$ & $1.554(1.135-2.129)$ & $0.006^{*}$ & $1.892(1.370-2.611)$ & $<0.001^{*}$ \\
\hline Sarcopenia & $2.540(1.855-3.423)$ & $<0.001^{*}$ & $2.295(1.662-3.170)$ & $<0.001^{*}$ \\
\hline Gender (male/female) & $1.136(0.819-1.576)$ & 0.445 & $1.063(0.756-1.496)$ & 0.725 \\
\hline Age $\geq 75 y$ & $1.744(1.268-2.398)$ & $0.001^{*}$ & $1.296(0.898-1.870)$ & 0.165 \\
\hline BMI $<18.5$ & $1.567(0.997-2.463)$ & 0.052 & $1.515(0.930-2.467)$ & 0.095 \\
\hline ASA $\geq \mathrm{III}$ & $1.620(1.176-2.232)$ & $0.003^{*}$ & $1.661(1.181-2.337)$ & $0.004^{*}$ \\
\hline Charlson comorbidity index $\geq 1$ & $1.056(0.777-1.437)$ & 0.726 & $1.240(0.904-1.701)$ & 0.183 \\
\hline Anemia & $1.790(1.355-2.363)$ & $<0.001^{*}$ & $1.892(1.408-2.543)$ & $<0.001^{*}$ \\
\hline Hypoproteinemia & $1.645(1.221-2.215)$ & 0.001 & $1.764(1.290-2.413)$ & $<0.001^{*}$ \\
\hline Previous abdominal surgery & $0.945(0.763-1.170)$ & 0.602 & $1.207(0.779-1.872)$ & 0.400 \\
\hline TNM stage & & $<0.001^{*}$ & & \\
\hline II/I & $2.627(1.494-4.620)$ & $0.001^{*}$ & $5.802(2.885-11.668)$ & $<0.001^{*}$ \\
\hline III/I & $8.502(5.270-13.717)$ & $<0.001^{*}$ & $14.994(7.875-28.549)$ & $<0.001^{*}$ \\
\hline Tumor differentiation Undifferentiated/Differentiated & $2.404(1.694-3.413)$ & $<0.001^{*}$ & $2.813(1.908-4.147)$ & $<0.001^{*}$ \\
\hline Tumor location at cardia & $1.191(0.803-1.766)$ & 0.384 & $1.145(0.750-1.745)$ & 0.531 \\
\hline Type of resection (Total gastrectomy/Subtotal gastrectomy & $2.096(1.586-2.776)$ & $<0.001^{*}$ & $2.121(1.577-2.853)$ & $<0.001^{*}$ \\
\hline Laparoscopic surgery & $0.453(0.306-0.672)$ & $<0.001^{*}$ & $0.504(0.338-0.751)$ & $0.001^{*}$ \\
\hline Combined organ resection & $1.904(1.252-2.896)$ & $0.003^{*}$ & $2.176(1.415-3.348)$ & $<0.001^{*}$ \\
\hline Extent of lymph node dissection (D2/D1) & $1.225(0.805-1.865)$ & 0.343 & $1.273(0.807-2.009)$ & 0.300 \\
\hline Adjuvant chemotherapy & $0.813(0.616-1.073)$ & 0.144 & $1.062(0.788-1.430)$ & 0.693 \\
\hline Postoperative complications & & $0.002^{*}$ & & 0.257 \\
\hline Grade II/ No & $1.592(1.146-2.210)$ & $0.006^{*}$ & $1.258(0.872-1.816)$ & 0.220 \\
\hline Grade III-V/ No & $1.887(1.166-3.054)$ & $0.010^{*}$ & $1.433(0.825-2.491)$ & 0.202 \\
\hline
\end{tabular}

*Statistically significant $(P<0.05)$;

HR, hazard Ratio; CI, confidence interval; BMI, body mass index; ASA, American Society of Anesthesiologists; TNM, tumor-node-metastasis. 
Table 5. Multivariate analysis for risk factors associated with overall survival

\begin{tabular}{|c|c|c|c|c|c|c|c|c|}
\hline & \multicolumn{2}{|l|}{ MUST $\geq 1$} & \multicolumn{2}{|l|}{ NRS-2002 $\geq 3$} & \multicolumn{2}{|l|}{ MST $\geq 2$} & \multicolumn{2}{|l|}{ SNAQ $\geq 2$} \\
\hline & $\mathrm{HR}(95 \% \mathrm{CI})$ & $P$ & HR $(95 \% \mathrm{CI})$ & $P$ & $\mathrm{HR}(95 \% \mathrm{CI})$ & $P$ & $\mathrm{HR}(95 \% \mathrm{CI})$ & $P$ \\
\hline Nutritional risk & $1.192(0.878-1.619)$ & 0.260 & $1.391(1.029-1.878)$ & $0.032^{*}$ & $1.159(0.859-1.564)$ & 0.335 & $1.007(0.852-1.189)$ & 0.937 \\
\hline Sarcopenia & $1.609(1.141-2.268)$ & $0.007^{*}$ & $1.562(1.108-2.201)$ & $0.011^{*}$ & $1.598(1.134-2.252)$ & $0.007^{*}$ & 1.605 (1.137-2.264) & $0.007^{*}$ \\
\hline Gender (male/female) & 1.189 (0.846-1.669) & 0.319 & $1.178(0.840-1.653)$ & 0.343 & $1.183(0.843-1.660)$ & 0.330 & $1.165(0.831-1.634)$ & 0.377 \\
\hline Age $\geq 75 y$ & $1.042(0.716-1.515)$ & 0.831 & $1.022(0.704-1.484)$ & 0.909 & $1.030(0.708-1.498)$ & 0.877 & $1.042(0.717-1.514)$ & 0.829 \\
\hline BMI $<18.5$ & $1.249(0.765-2.039)$ & 0.373 & $1.209(0.748-1.954)$ & 0.439 & $1.308(0.812-2.106)$ & 0.270 & 1.355 (0.843-2.178) & 0.209 \\
\hline ASA $\geq \mathrm{III}$ & $1.129(0.804-1.586)$ & 0.485 & $1.110(0.789-1.562)$ & 0.548 & $1.118(0.796-1.570)$ & 0.520 & $1.118(0.796-1.569)$ & 0.520 \\
\hline Anemia & $1.071(0.779-1.474)$ & 0.672 & 1.079 (0.785-1.482) & 0.640 & $1.073(0.779-1.476)$ & 0.668 & 1.083 (0.787-1.491) & 0.624 \\
\hline Hypoproteinemia & $0.882(0.624-1.246)$ & 0.475 & 0.847 (0.599-1.197) & 0.346 & $0.904(0.642-1.275)$ & 0.566 & $0.904(0.639-1.277)$ & 0.566 \\
\hline Undifferentiated Histologic type & $1.532(1.058-2.218)$ & $0.024^{*}$ & $1.528(1.056-2.211)$ & $0.025^{*}$ & $1.549(1.070-2.240)$ & $0.020^{*}$ & $1.544(1.067-2.234)$ & $0.021^{*}$ \\
\hline TNM stage & & $<0.001^{*}$ & & $<0.001^{*}$ & & $<0.001^{*}$ & & \\
\hline II/I & $2.623(1.438-4.784)$ & $0.002^{*}$ & $2.619(1.437-4.776)$ & $0.002^{*}$ & $2.638(1.446-4.812)$ & $0.002^{*}$ & $2.688(1.475-4.898)$ & $0.001^{*}$ \\
\hline III/I & $8.059(4.736-13.711)$ & $<0.001$ & $8.265(4.865-14.040)$ & $<0.001^{*}$ & $8.131(4.783-13.821)$ & $<0.001^{*}$ & $8.306(4.889-14.114)$ & $<0.001^{*}$ \\
\hline Total gastrectomy & 1.569 (1.173-2.098) & $0.002^{*}$ & $1.566(1.171-2.094)$ & $0.002^{*}$ & $1.571(1.175-2.101)$ & $0.002^{*}$ & $1.589(1.189-2.124)$ & $0.002^{*}$ \\
\hline Laparoscopic surgery & $0.888(0.588-1.342)$ & 0.574 & $0.915(0.605-1.386)$ & 0.676 & $0.878(0.582-1.326)$ & 0.536 & $0.877(0.580-1.325)$ & 0.533 \\
\hline Combined resection & $1.257(0.812-1.945)$ & 0.305 & $1.252(0.809-1.936)$ & 0.313 & $1.219(0.786-1.892)$ & 0.377 & $1.249(0.808-1.932)$ & 0.317 \\
\hline Postoperative complications & & 0.358 & & 0.333 & & 0.335 & & 0.315 \\
\hline Grade II/ No & $1.218(0.863-1.719)$ & 0.262 & $1.221(0.865-1.724)$ & 0.255 & $1.230(0.872-1.735)$ & 0.239 & $1.231(0.872-1.736)$ & 0.238 \\
\hline Grade III-V/No & $1.349(0.805-2.259)$ & 0.256 & $1.370(0.818-2.293)$ & 0.231 & $1.352(0.808-2.263)$ & 0.251 & $1.376(0.819-2.309)$ & 0.228 \\
\hline Adjuvant chemotherapy & $0.444(0.326-0.603)$ & $<0.001^{*}$ & $0.436(0.321-0.594)$ & $<0.001^{*}$ & $0.440(0.323-0.599)$ & $<0.001^{*}$ & $0.444(0.326-0.605)$ & $<0.001^{*}$ \\
\hline
\end{tabular}

*Statistically significant $(P<0.05)$;

HR, hazard Ratio; CI, confidence interval; BMI, body mass index; ASA, American Society of Anesthesiologists; TNM, tumor-node-metastasis.

Table 6. Multivariate analysis for risk factors associated with disease-free survival

\begin{tabular}{|c|c|c|c|c|c|c|c|c|}
\hline & MUST $\geq 1$ & & NRS-2002 $\geq 3$ & & $\mathrm{MST} \geq 2$ & & $\mathrm{SNAQ} \geq 2$ & \\
\hline & HR $(95 \% \mathrm{CI})$ & $P$ & HR $(95 \% \mathrm{CI})$ & $P$ & HR $(95 \% \mathrm{CI})$ & $P$ & HR $(95 \% \mathrm{CI})$ & $P$ \\
\hline Nutritional risk & $0.936(0.678-1.292)$ & 0.687 & $0.853(0.615-1.184)$ & 0.342 & 1.009 (0.733-1.389) & 0.956 & $0.893(0.755-1.056)$ & 0.186 \\
\hline Sarcopenia & $1.611(1.121-2.314)$ & $0.011^{*}$ & $1.636(1.136-2.356)$ & $0.008^{*}$ & 1.609 (1.119-2.312) & $0.010^{*}$ & $1.579(1.097-2.274)$ & $0.014^{*}$ \\
\hline Gender (male/female) & $1.033(0.726-1.470)$ & 0.855 & $1.039(0.732-1.475)$ & 0.831 & $1.042(0.733-1.482)$ & 0.817 & $1.060(0.746-1.506)$ & 0.746 \\
\hline Age $\geq 75 y$ & $0.732(0.480-1.118)$ & 0.149 & $0.738(0.483-1.128)$ & 0.160 & $0.731(0.478-1.118)$ & 0.149 & 0.725 (0.474-1.109) & 0.139 \\
\hline $\mathrm{BMI}<18.5$ & $1.524(0.897-2.591)$ & 0.119 & $1.561(0.927-2.629)$ & 0.094 & $1.474(0.885-2.456)$ & 0.136 & $1.454(0.876-2.414)$ & 0.148 \\
\hline ASA $\geq \mathrm{III}$ & $1.351(0.946-1.929)$ & 0.098 & $1.353(0.948-1.931)$ & 0.096 & $1.356(0.950-1.936)$ & 0.094 & $1.356(0.949-1.937)$ & 0.094 \\
\hline Anemia & $1.153(0.823-1.614)$ & 0.408 & $1.153(0.823-1.614)$ & 0.408 & 1.149 (0.821-1.609) & 0.418 & $1.142(0.815-1.599)$ & 0.440 \\
\hline Hypoproteinemia & $1.011(0.701-1.458)$ & 0.954 & 1.029 (0.711-1.488) & 0.880 & $1.002(0.697-1.441)$ & 0.992 & $0.971(0.674-1.400)$ & 0.876 \\
\hline Undifferentiated Histologic type & $1.727(1.154-2.585)$ & $0.008^{*}$ & $1.734(1.159-2.594)$ & $0.007^{*}$ & $1.720(1.150-2.573)$ & $0.008^{*}$ & $1.714(1.146-2.563)$ & $0.009^{*}$ \\
\hline TNM stage & & $<0.001^{*}$ & & $<0.001^{*}$ & & $<0.001^{*}$ & & $<0.001^{*}$ \\
\hline II/I & $6.180(2.978-12.824)$ & $<0.001^{*}$ & $6.199(2.990-12.850)$ & $<0.001^{*}$ & $6.122(2.951-12.702)$ & $<0.001^{*}$ & $6.063(2.923-12.573)$ & $<0.001^{*}$ \\
\hline III/I & $14.374(7.235-28.588)$ & $<0.001^{*}$ & $14.271(7.192-28.297)$ & $<0.001^{*}$ & $14.215(7.157-28.234)$ & $<0.001^{*}$ & $13.915(7.007-27.633)$ & $<0.001^{*}$ \\
\hline Total gastrectomy & $1.530(1.124-2.082)$ & $0.007^{*}$ & $1.528(1.124-2.078)$ & $0.007^{*}$ & $1.521(1.118-2.070)$ & $0.008^{*}$ & $1.517(1.116-2.063)$ & $0.008^{*}$ \\
\hline Laparoscopic surgery & $0.958(0.631-1.453)$ & 0.839 & $0.941(0.619-1.430)$ & 0.775 & $0.961(0.634-1.457)$ & 0.851 & $0.974(0.642-1.478)$ & 0.902 \\
\hline Combined resection & $1.459(0.939-2.267)$ & 0.093 & $1.458(0.939-2.264)$ & 0.093 & $1.466(0.943-2.279)$ & 0.089 & $1.489(0.958-2.315)$ & 0.077 \\
\hline Postoperative complications & & 0.952 & & 0.952 & & 0.957 & & 0.992 \\
\hline Grade II/ No & $0.983(0.671-1.438)$ & 0.928 & $0.984(0.673-1.440)$ & 0.935 & $0.978(0.669-1.431)$ & 0.910 & $0.977(0.667-1.430)$ & 0.905 \\
\hline Grade III-V/No & $1.086(0.605-1.950)$ & 0.783 & $1.087(0.606-1.950)$ & 0.778 & $1.076(0.598-1.935)$ & 0.807 & $1.004(0.551-1.826)$ & 0.991 \\
\hline Adjuvant chemotherapy & $1.727(1.154-2.585)$ & $0.008^{*}$ & $0.474(0.342-0.658)$ & $<0.001^{*}$ & $0.471(0.340-0.654)$ & $<0.001^{*}$ & $0.465(0.335-0.645)$ & $<0.001^{*}$ \\
\hline
\end{tabular}

*Statistically significant $(P<0.05)$;

HR, hazard Ratio; CI, confidence interval; BMI, body mass index; ASA, American Society of Anesthesiologists; TNM, tumor-node-metastasis.

Although the 4 nutritional screening tools showed a significant correlation with sarcopenia, their agreements with sarcopenia was low (kappa $<0.2$ ), because they focus on different aspects in their components of definition compared with sarcopenia. Generally, these nutritional screening tools mainly focus on the BMI, weight loss or reduced intake of food. However, none of these tools distinguish the body compositions. Different body compositions have distinct impact on the surgical outcomes. Low muscle mass has been recognized to be associated with poor clinical outcomes $[19,20]$. However, visceral obesity has been found to be a risk factor for postoperative complications [21, 22]. Sarcopenic obesity, which is characterized by low muscle mass and high BMI or high visceral fat mass, was associated with negative clinical outcomes [23, 24]. However, these patients cannot be identified by traditional nutritional screening tools. One important feature of sarcopenia is the loss of muscle mass. Therefore, measurement of sarcopenia could add significant information for risk assessment before surgery. Moreover, physical function has been increasing recognized to be associated with adverse postoperative outcomes. 
Reddy et al. reported that timed stair climbing is the single strongest predictor of perioperative complications in patients undergoing abdominal surgery, even better than the ACS NSQIP calculator [25]. Sato et al. showed that low hand grip strength was a significant risk factor for morbidity after gastric cancer surgery [26]. Physical function is another important component of sarcopenia, which can also explain the additional predictive value of sarcopenia over nutritional screening tools. In the present study, we used the revised European consensus on definition and diagnosis of sarcopenia recommended by EWGSOP2 [4]. Compared with the previous consensus of sarcopenia definition recommended by EWGSOP1 [27], the revised consensus added low muscle quality to the diagnosis of sarcopenia. Muscle quality is a relatively new term, referring to the changes in muscle architecture and composition [28]. Low muscle quality, as measured by the attenuation of the muscle has been proved to have significant influence on the prognosis after surgery [17]. This can also explain the superior predictive value of sarcopenia in our study.

In conclusion, our study demonstrated that none of the 4 nutritional screening tools have strong predictive power for postoperative complications and long-term survival in patients underwent radical gastrectomy for gastric cancer. Sarcopenia had additional predictive value for postoperative complications and long-term survival besides these nutritional screening tools. Our study demonstrated the superior predictive value of sarcopenia over nutritional screening tools for the prediction of postoperative outcomes. Therefore, measurement of sarcopenia is necessary for optimizing the preoperative risk assessment for gastric cancer surgery, even when the nutritional screening has already been well implemented.

\section{Abbreviations}

EWGSOP: European Working Group on Sarcopenia in Older People; CT: computed tomography; MUST: Malnutrition Universal Screening Tool; NRS-2002: Nutrition Risk Screening 2002; MST: Malnutrition Screening Tool; SNAQ: Short Nutritional Assessment Questionnaire; ASA: American Society of Anesthesiologists; BMI: body mass index; TNM: tumor-node-metastasis; L3: third lumbar vertebra; HU: Hounsfield units; SMI: skeletal muscle index; OS: overall survival; DFS: disease-free survival; SD: standard deviation; IQR: interquartile range; OR: odds ratio; HR: hazard Ratio; CI: confidence interval; ROC: receiver operating characteristic.

\section{Acknowledgements}

This study was funded by the National Natural Science Foundation of China (No. 81770884), Zhejiang Provincial Health Department Medical Support Discipline-Nutrition (11-ZC24), and the Youth Program of Science and Technology Innovation Foundation of Wenzhou Medical University (KYYW201921).

\section{Supplementary Material}

Supplementary figures and tables. http://www.jcancer.org/v11p5852s1.pdf

\section{Authors' contribution}

Qian-Tong Dong and Xiao-Lei Chen designed the study. Hui-Yang Cai, Drugyel Wangchuk, and Xi-Yi Chen collected the data. Dong-Dong Huang, Wen-Xi Dong and Jing-Yi Yan did the analysis and interpretation of data. Dong-Dong Huang wrote the article. Xiao-Lei Chen revised the article and took the decision to submit the article for publication.

\section{Compliance with ethical standards}

This study was approved by the Ethics Committee of The First Affiliated Hospital of Wenzhou Medical University.

\section{Human rights statement and informed consent}

All procedures followed were in accordance with the ethical standards of the Ethics Committee of The First Affiliated Hospital of Wenzhou Medical University and with the Helsinki Declaration of 1964 and later versions. Informed consent to be included in the study was obtained from all patients.

\section{Competing Interests}

The authors have declared that no competing interest exists.

\section{References}

1. Huang DD, Zhou CJ, Wang SL, Mao ST, Zhou XY, Lou N, et al. Impact of different sarcopenia stages on the postoperative outcomes after radical gastrectomy for gastric cancer. Surgery. 2017; 161: 680-93.

2. Huang DD, Chen $X X$, Chen $X Y$, Wang SL, Shen $X$, Chen $X L$, et al. Sarcopenia predicts 1 -year mortality in elderly patients undergoing curative gastrectomy for gastric cancer: a prospective study. Journal of cancer research and clinical oncology. 2016; 142: 2347-56.

3. Huang DD, Wang SL, Zhuang CL, Zheng BS, Lu JX, Chen FF, et al. Sarcopenia, as defined by low muscle mass, strength and physical performance, predicts complications after surgery for colorectal cancer. Colorectal disease : the official journal of the Association of Coloproctology of Great Britain and Ireland. 2015; 17: O256-64.

4. Cruz-Jentoft AJ, Bahat G, Bauer J, Boirie Y, Bruyere O, Cederholm T, et al. Sarcopenia: revised European consensus on definition and diagnosis. Age and ageing. 2019; 48: 16-31.

5. van der Kroft G, Bours D, Janssen-Heijnen DM, van Berlo D, Konsten D. Value of sarcopenia assessed by computed tomography for the prediction of postoperative morbidity following oncological colorectal resection: A comparison with the malnutrition screening tool. Clinical nutrition ESPEN. 2018; 24: 114-9. 
6. Kondrup J, Allison SP, Elia M, Vellas B, Plauth M, et al. ESPEN guidelines for nutrition screening 2002. Clinical nutrition (Edinburgh, Scotland). 2003; 22: 415-21.

7. Arends J, Bachmann P, Baracos V, Barthelemy N, Bertz H, Bozzetti F, et al. ESPEN guidelines on nutrition in cancer patients. Clinical nutrition (Edinburgh, Scotland). 2017; 36: 11-48.

8. Fujiya K, Kawamura T, Omae K, Makuuchi R, Irino T, Tokunaga M, et al. Impact of Malnutrition After Gastrectomy for Gastric Cancer on Long-Term Survival. Ann Surg Oncol. 2018; 25: 974-83.

9. Schwegler I, von Holzen A, Gutzwiller JP, Schlumpf R, Muhlebach S, Stanga Z. Nutritional risk is a clinical predictor of postoperative mortality and morbidity in surgery for colorectal cancer. The British journal of surgery. 2010; 97: 92-7.

10. Stratton RJ, Hackston A, Longmore D, Dixon R, Price S, Stroud M, et al. Malnutrition in hospital outpatients and inpatients: prevalence, concurrent validity and ease of use of the 'malnutrition universal screening tool' ('MUST') for adults. The British journal of nutrition. 2004; 92: 799-808.

11. Kondrup J, Rasmussen $\mathrm{HH}$, Hamberg O, Stanga $\mathrm{Z}$. Nutritional risk screening (NRS 2002): a new method based on an analysis of controlled clinical trials. Clinical nutrition (Edinburgh, Scotland). 2003; 22: 321-36.

12. Ferguson M, Capra S, Bauer J, Banks M. Development of a valid and reliable malnutrition screening tool for adult acute hospital patients. Nutrition (Burbank, Los Angeles County, Calif). 1999; 15: 458-64.

13. Kruizenga HM, Seidell JC, de Vet HC, Wierdsma NJ, van Bokhorst-de van der Schueren MA. Development and validation of a hospital screening tool for malnutrition: the short nutritional assessment questionnaire (SNAQ). Clinical nutrition (Edinburgh, Scotland). 2005; 24: 75-82.

14. Japanese gastric cancer treatment guidelines 2010 (ver. 3). Gastric Cancer. 2011; 14: 113-23.

15. Dindo D, Demartines N, Clavien PA. Classification of surgical complications: a new proposal with evaluation in a cohort of 6336 patients and results of a survey. Ann Surg. 2004; 240: 205-13.

16. Peng P, Hyder O, Firoozmand A, Kneuertz P, Schulick RD, Huang D, et al. Impact of sarcopenia on outcomes following resection of pancreatic adenocarcinoma. Journal of gastrointestinal surgery : official journal of the Society for Surgery of the Alimentary Tract. 2012; 16: 1478-86

17. Zhuang $C L$, Shen $X$, Huang $Y Y$, Zhang FM, Chen XY, Ma LL, et al. Myosteatosis predicts prognosis after radical gastrectomy for gastric cancer: A propensity score-matched analysis from a large-scale cohort. Surgery. 2019; 166: 297-304.

18. Ma BW, Chen XY, Fan SD, Zhang FM, Huang DD, Li B, et al. Impact of sarcopenia on clinical outcomes after radical gastrectomy for patients without nutritional risk. Nutrition (Burbank, Los Angeles County, Calif). 2019; 61: 61-6.

19. van Vledder MG, Levolger S, Ayez N, Verhoef C, Tran TC, Ijzermans JN. Body composition and outcome in patients undergoing resection of colorectal liver metastases. The British journal of surgery. 2012; 99: 550-7.

20. Harimoto N, Shirabe K, Yamashita YI, Ikegami T, Yoshizumi T, Soejima Y, et al. Sarcopenia as a predictor of prognosis in patients following hepatectomy for hepatocellular carcinoma. The British journal of surgery. 2013; 100: 1523-30.

21. Wang SL, Ma LL, Chen XY, Zhou DL, Li B, Huang DD, et al. Impact of visceral fat on surgical complications and long-term survival of patients with gastric cancer after radical gastrectomy. European journal of clinical nutrition. 2018; 72: 436-45.

22. Chen WZ, Chen XD, Ma LL, Zhang FM, Lin J, Zhuang CL, et al. Impact of Visceral Obesity and Sarcopenia on Short-Term Outcomes After Colorectal Cancer Surgery. Digestive diseases and sciences. 2018; 63: 1620-30.

23. Kamo N, Kaido T, Hamaguchi Y, Okumura S, Kobayashi A, Shirai H, et al. Impact of sarcopenic obesity on outcomes in patients undergoing living donor liver transplantation. Clinical nutrition (Edinburgh, Scotland). 2019; 38: 2202-9.

24. Ratnayake CBB, Wells C. Sarcopenic obesity and post-operative morbidity after pancreatic surgery: a cohort study. ANZ J Surg. 2019; 89: 1587-92.

25. Reddy S, Contreras CM Singletary B, Bradford TM, Waldrop MG, Mims AH, et al. Timed Stair Climbing Is the Single Strongest Predictor of Perioperative Complications in Patients Undergoing Abdominal Surgery. J Am Coll Surg. 2016; 222: 559-66.

26. Sato T, Aoyama T, Hayashi T, Segami K, Kawabe T, Fujikawa H, et al. Impact of preoperative hand grip strength on morbidity following gastric cancer surgery. Gastric Cancer. 2016; 19: 1008-15.

27. Cruz-Jentoft AJ, Baeyens JP, Bauer JM, Boirie $\mathrm{Y}$, Cederholm $\mathrm{T}$, Landi $\mathrm{F}$, et al Sarcopenia: European consensus on definition and diagnosis: Report of the European Working Group on Sarcopenia in Older People. Age and ageing. 2010; 39: 412-23.

28. McGregor RA, Cameron-Smith D, Poppitt SD. It is not just muscle mass: a review of muscle quality, composition and metabolism during ageing as determinants of muscle function and mobility in later life. Longevity \& healthspan. $2014 ; 3: 9$. 